\title{
Spectroscopic trace of the Lifshitz transition and multivalley activation in thermoelectric SnSe under high pressure
}

\author{
Tobias Biesner $\mathbb{1}^{1}$, Weiwu Li $\mathbb{1}^{1}$, Alexander A. Tsirlin $\mathbb{E}^{2}$, Seulki Roh${ }^{1}$, Pai-Chun Wei $\mathbb{D}^{3,4}$, Ece Uykur ${ }^{1}$ and \\ Martin Dressel (1)
}

\begin{abstract}
Multivalley systems offer not only exciting physical phenomena but also the possibility of broad utilization. Identifying an important platform and understanding its physics are paramount tasks to improve their capability for application. Here, we investigate a promising candidate, the semiconductor SnSe, by optical spectroscopy and density functional theory calculations. Upon applying pressure to lightly doped SnSe, we directly monitored the phase transition from semiconductor to semimetal. In addition, heavily doped SnSe exhibited a successive Lifshitz transition, activating multivalley physics. Our comprehensive study provides insight into the effects of pressure and doping on this system, leading to promising routes to tune the material properties for advanced device applications, including thermoelectrics and valleytronics.
\end{abstract}

\section{Introduction}

Multivalley electronic systems confining charge carriers to a specific momentum have emerged as a key aspect of new technologies ${ }^{1,2}$. These systems have a unique band structure that consists of multiple valence band maxima and conduction band minima with different momenta near the Fermi energy level, providing specific optoelectronic properties ${ }^{3}$. The distinguished momentum dependence in such systems provides an additional degree of freedom for information processing, opening an avenue for valleytronics ${ }^{4,5}$. On the other hand, the multivalley effect improves the efficiency of thermoelectric materi$\mathrm{als}^{6,7}$. In particular, the Seebeck coefficient can be tuned by increasing the band degeneracy number and the effective mass with an increasing number of valleys ${ }^{8-10}$. In

Correspondence: Tobias Biesner (tobias.biesner@pi1.physik.uni-stuttgart.de) or Weiwu Li (Iww60253496@sina.com) or Pai-Chun Wei (pcwei68@ntu.edu.tw)

11. Physikalisches Institut, Universität Stuttgart, Stuttgart, Germany

${ }^{2}$ Experimental Physics VI, Center for Electronic Correlations and Magnetism,

University of Augsburg, Augsburg, Germany

Full list of author information is available at the end of the article addition to identifying accessible multivalley systems, it is highly desirable to develop proper ways to tune them.

The semiconductor SnSe is a promising multivalley system and is known for its outstanding thermoelectric properties ${ }^{11-15}$. In $\mathrm{SnSe}$, the strong electronic anisotropy with multiple valence band maxima and conduction band minima induces two distinct gaps along the $\Gamma-Y$ and $\Gamma-$ $Z$ directions of the Brillouin zone, corresponding to the zigzag and armchair anisotropy, respectively (see Fig. 1a for the crystal structure) $)^{3,11,16-21}$. To further promote multivalley physics, chemical doping was performed to push the Fermi level deep into the valence band, activating multiple hole pockets (Fig. 1b) ${ }^{6,16,19,21-23}$. Furthermore, samples were unintentionally self-doped by $\mathrm{Sn}$ vacancies or $\mathrm{SnSe}_{2}$ microdomains, resulting in a small shift in the Fermi level that caused the resistivity of heavily doped SnSe to deviate from its semiconducting behavior, generating a metallic temperature dependence, as depicted in Fig. $1 \mathrm{c}^{17,24}$.

Pressure is a clean tuning parameter for manipulating material properties ${ }^{25}$. In heavily doped SnSe, a pressureinduced Lifshitz transition occurs, i.e., a change in the Fermi surface topology without a structural transition ${ }^{26}$. At

\section{(c) The Author(s) 2021}

(c) Open Access This article is licensed under a Creative Commons Attribution 4.0 International License, which permits use, sharing, adaptation, distribution and reproduction cc) in any medium or format, as long as you give appropriate credit to the original author(s) and the source, provide a link to the Creative Commons license, and indicate if changes were made. The images or other third party material in this article are included in the article's Creative Commons license, unless indicated otherwise in a credit line to the material. If material is not included in the article's Creative Commons license and your intended use is not permitted by statutory regulation or exceeds the permitted use, you will need to obtain permission directly from the copyright holder. To view a copy of this license, visit http://creativecommons.org/licenses/by/4.0/. 


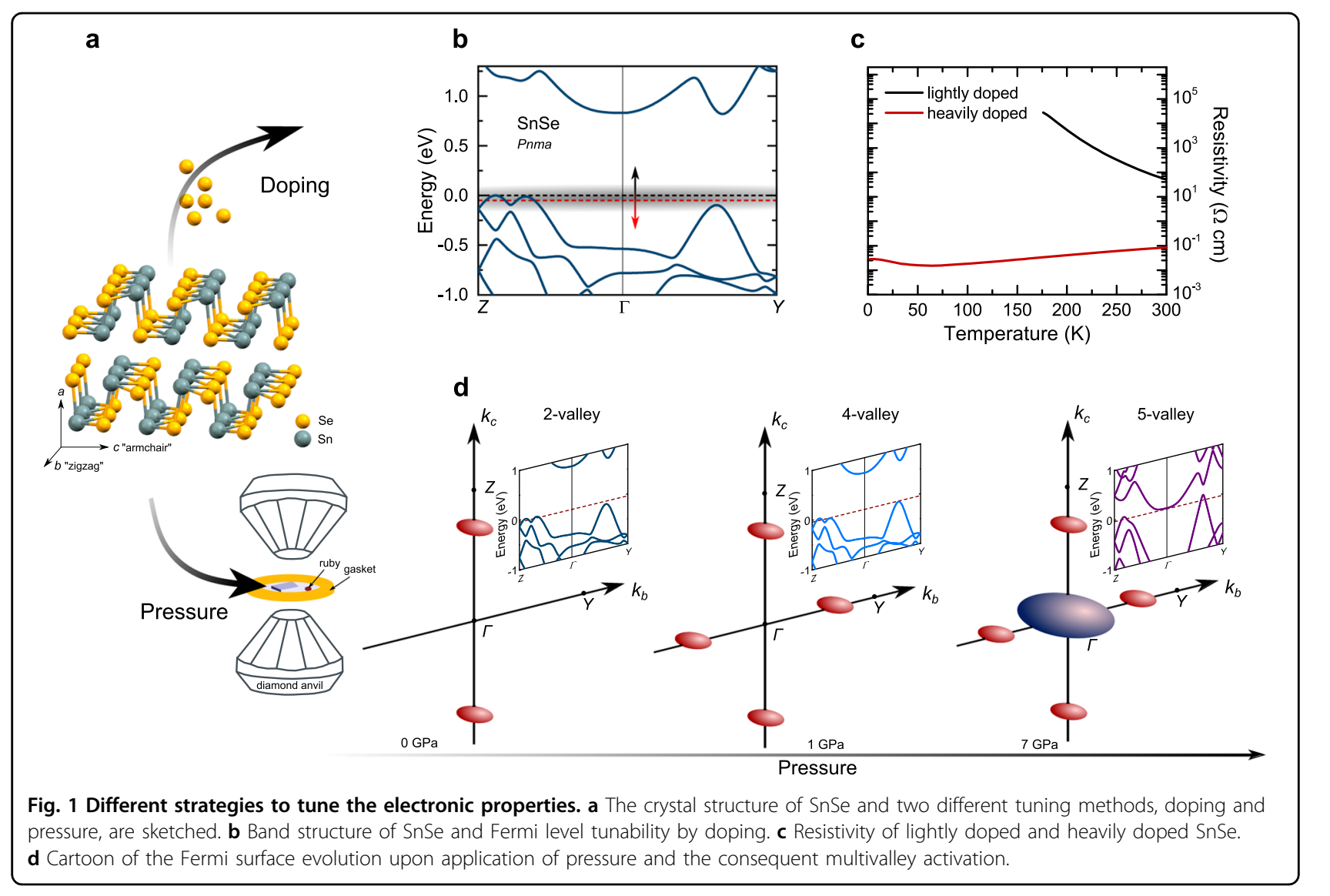

$0.86 \mathrm{GPa}$, the compound transforms from a two-valley system to a four-valley system, accompanied by improved thermoelectric properties, providing proof that pressure is a powerful tool to tune the electronic multivalley ${ }^{27,28}$. Densityfunctional theory (DFT) calculations and transport studies have reported enhanced thermoelectric properties of $\mathrm{SnSe}$ upon application of external pressure ${ }^{29-32}$. Although transport measurements ${ }^{33,34}$ and theoretical approaches ${ }^{35,36}$ provide evidence for a semiconductor-semimetal transition, direct investigations of the electronic structure and its pressure dependence are still lacking. In fact, transport measurements are confined to a cumulative probe of the conduction bands without spectroscopic resolution.

In this study, we employ pressure-dependent infrared spectroscopy to directly map the multivalley electronic band structure of $\mathrm{SnSe}$ over a wide pressure range up to $12 \mathrm{GPa}$ in lightly doped and heavily doped samples. By combining these experiments with first-principles calculations, we explain the electronic structure evolution of SnSe from semiconductor-to-semimetal under pressure. In addition to the results from transport studies, in heavily doped SnSe, we observe a Lifshitz transition in the highpressure range above $7 \mathrm{GPa}$, where a new electron pocket emerges (see the sketched Fermi surface in Fig. 1d). Our study provides a comprehensive insight into SnSe under pressure, suggesting strategies to tune the optoelectronic and thermoelectric properties to allow device applications in more efficient ways.

\section{Materials and methods}

Sample preparation and resistivity measurements

Two $<100>$-cleaved SnSe samples (S1 and S2) with different carrier concentrations (lightly doped and heavily doped) were prepared by the Bridgman method ${ }^{24}$. The carrier concentrations (resistivity) of sample S1 and sample S2 were measured by the Quantum Design ${ }^{\circledR}$ Physical Properties Measurement System (PPMS) to be $3 \times 10^{15} \mathrm{~cm}^{-3}(50.5 \Omega \mathrm{cm})$ and $5 \times 10^{17} \mathrm{~cm}^{-3}(0.086 \Omega$ $\mathrm{cm})$ at $300 \mathrm{~K}$, respectively. With decreasing temperature, sample S1 showed semiconducting-like behavior, whereas sample S2 showed metallic-like behavior. The size of the two samples used for the pressure-dependent optical measurements was approximately 180 (length) $\times 160$ (width) $\times 40 \mu \mathrm{m}$ (thickness). Further information about the sample homogeneity can be found in the supplemental material (Fig. S1)

\section{Optical measurements under high pressure}

A screw-driven diamond anvil cell (Almax easyLab) with Type IIa diamonds and an $800 \mu \mathrm{m}$ culet was used. 


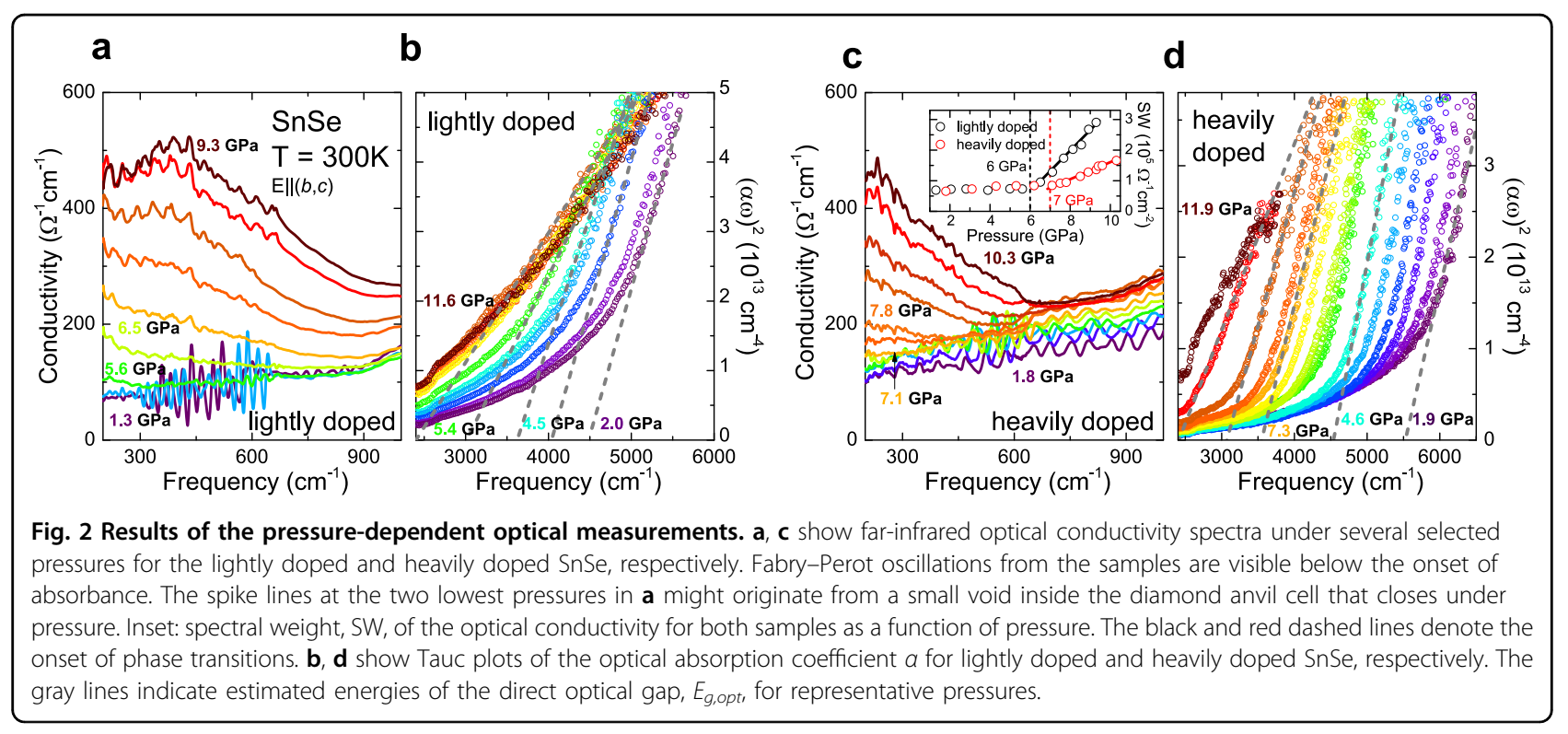

$\mathrm{CuBe}$ gaskets with a thickness of $60 \mu \mathrm{m}$ and a hole size of $\sim 250 \mu \mathrm{m}$ were prepared for sample space, leaving enough free area for multiple ruby spheres, which were used for pressure calibration $^{37,38}$ (cf. Fig. S2). CsI was utilized as a quasi-hydrostatic pressure transmitting medium. Reflectance and transmittance spectra were measured by employing a Fourier transform spectrometer (Bruker Vertex $80 \mathrm{v}$ ) under near-normal incidence, $E \|(b, c)$ at $T=$ $300 \mathrm{~K}$. For the reflectance measurements, we used the $\mathrm{CuBe}$ gasket as a reference and corrected the final spectra by the reflectance of CuBe. The transmittance was measured by referencing the transparent pressure media inside the diamond anvil cell. The optical conductivity was determined by modified Kramers-Kronig analysis of the reflectance, as described in the supplemental material. From the data in the mid-infrared range, we calculated the optical absorption coefficient as follows: $\alpha=\frac{1}{d} \ln \left\{\frac{(1-R)^{2}}{T r}\right\}^{39}$, where $R$ and $\operatorname{Tr}$ are the reflectance and transmittance, respectively, and $d$ is the sample thickness. Direct optical interband transitions, $E_{g, o p t}$, were determined using the Tauc method ${ }^{40}$ by extrapolating the linear regime of $(\alpha \omega)^{2}$. The spectral weight, SW, was determined by integrating the optical conductivity, $\sigma_{1}(\omega)$, over the frequency, $S W=\int_{\omega_{\min }}^{\omega_{\max }} \sigma_{1}(\omega) \mathrm{d} \omega$, and was directly related to the carrier density and effective mass (see the supplemental material for further information) ${ }^{40-42}$. Extended data sets and further details of the experimental analysis can be found in Figs. S3-S8.

\section{DFT calculations}

Relativistic density-functional band structure calculations were performed in the Wien2K $\operatorname{code}^{43}$ with the modified Becke-Johnson (mBJ) exchange-correlation functional $^{44}$ parametrized according to Ref. ${ }^{45}$. This approach delivers an accurate bandgap for $\mathrm{SnSe}$ at ambient pressure and correctly reproduces the pressure of the semiconductor-to-metal transition. In contrast, standard DFT functionals, such as the widely used PerdewBurke-Ernzerhof exchange-correlation potential ${ }^{46}$, tend to underestimate the ambient pressure bandgap and, consequently, the critical pressure where $\mathrm{SnSe}$ becomes metallic. Atomic positions from Ref. ${ }^{47}$ were used. The band structure and density of states were computed on a well-converged $k$ mesh with 1728 points in the irreducible part of the first Brillouin zone.

\section{Results}

\section{Optical spectra under pressure}

The optical conductivity of the lightly doped SnSe in the far-infrared range upon application of pressure is displayed in Fig. 2a. At ambient pressure, SnSe exhibits a phononic feature at low frequencies, which is also visible in our low-pressure spectra (see Fig. S9). At low pressures, we observe an insulating characteristic without pronounced free charge carriers, i.e., no Drude response ${ }^{40,42}$. With increasing pressure, the low-frequency optical conductivity is increasing with the development of a Drude mode when the pressure exceeds $6 \mathrm{GPa}$. In addition, the interband contribution changes with increasing pressure, as displayed in Fig. 2b. In particular, the direct optical gap is suppressed continuously, resulting in a redshift. However, at all pressures up to the highest pressure available $(11.6 \mathrm{GPa})$, the gap does not close completely, indicating that the metallization of SnSe above $6 \mathrm{GPa}$ is caused by a band shift across the Fermi level rather than a collapse of the optical gap owing to high pressure. The heavily doped sample (cf. Fig. 2c, d) yields similar results; however, in this 


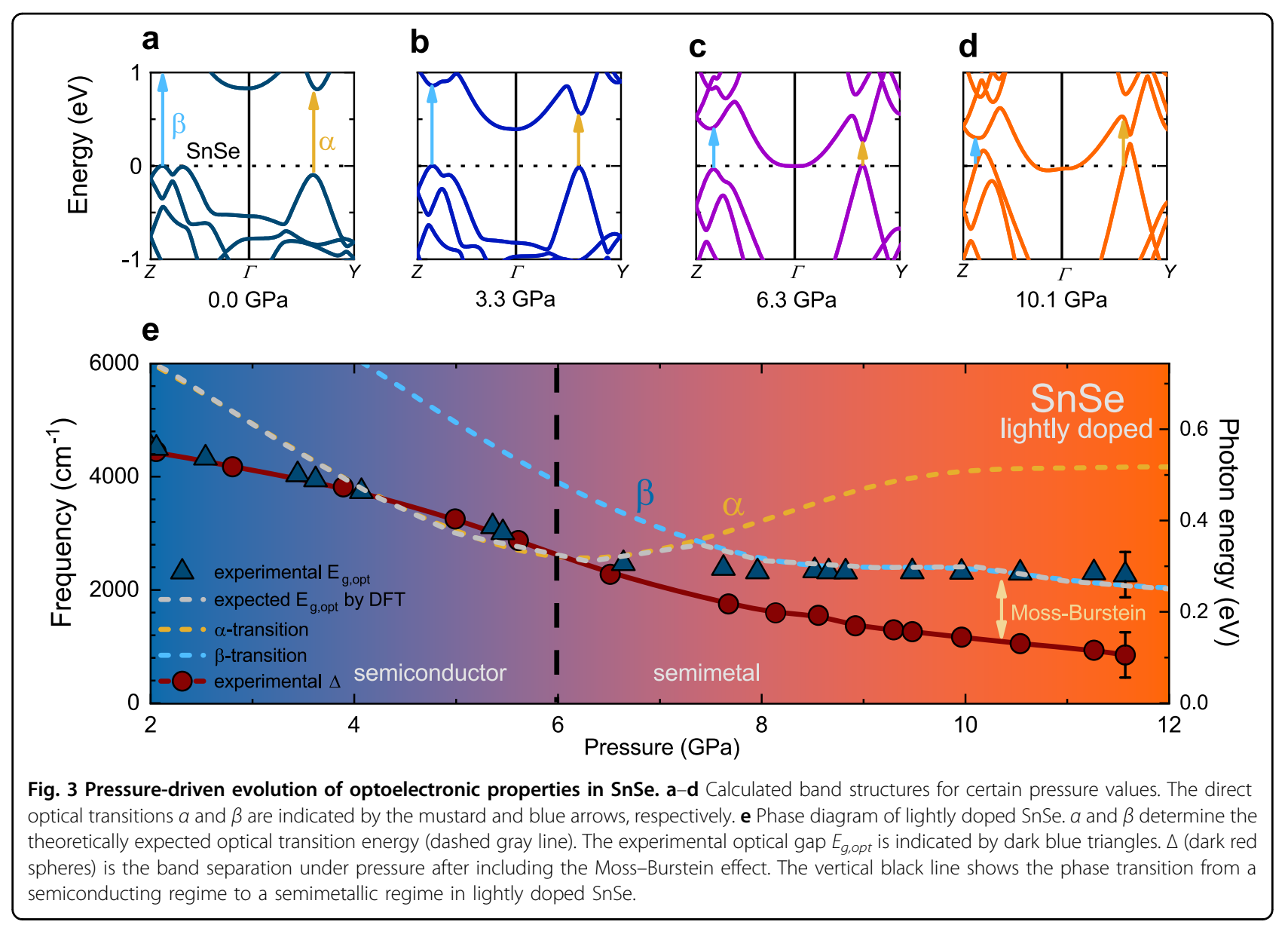

case, a delayed transition above $7 \mathrm{GPa}$ is observed. This delay is most visible in the $S W$ of the low-frequency range (inset of Fig. 2c), where the SW increases more strongly at an earlier pressure for the lightly doped SnSe.

\section{Optoelectronic properties under pressure}

To further scrutinize the electronic evolution under pressure, we compare the experimental results with the results of DFT calculations (Fig. 3a-d). The results along the $Z-\Gamma-Y$ path of the Brillouin zone are shown for selected pressures. In accordance with the ARPES measurements ${ }^{17}$ and previous calculations ${ }^{31,36}$, we identify multiple valence band maxima in the $\Gamma-Z$ and $\Gamma-Y$ directions and a minimum of the conduction band at the $\Gamma$ point, characteristic of the multivalley electronic structure of SnSe. In Fig. 3a, two distinct direct interband transitions, $\alpha$ and $\beta$, are most pronounced. In this case, the transition with lower energy determines the experimental optical gap, $E_{g o p t}$. Thus, at ambient pressure, in the semiconducting phase, $E_{g, o p t}$ is governed by transitions along the $\Gamma-Y$ path, $\alpha$.

As the pressure increases, the valence and conduction bands approach the Fermi energy level, $E_{F}$ (Fig. 3b), and eventually reach $E_{F}$ at $6.3 \mathrm{GPa}$ (Fig. 3c), rendering a semimetallic picture of the high-pressure phase. Furthermore, above $7 \mathrm{GPa}$, the optical transition energy $\beta$ becomes smaller than $\alpha$ (see Fig. 3d) and determines the value of $E_{g}$ ${ }_{\text {opt }}$. As the valence band penetrates through $E_{F}$, the interband transition differs from the band separation, $\Delta$; the Moss-Burstein effect ${ }^{48,49}, \Delta E_{g}^{B M}$, occurs. The band separation, $\Delta$, can thus be estimated from the experimental value of $E_{g, o p t}$ by correcting for $\Delta E_{g}^{B M}, \Delta=E_{g, o p t}-\Delta E_{g}^{B M}$.

In Fig. 3e, we summarize the results of the DFT calculations $(\alpha$ and $\beta$ ) and determine the theoretically expected optical gap. From Fig. 2b, we extract the experimentally observed transition energy. To obtain the experimental band separation, we correct the optical gap for the Moss-Burstein effect by using the SW of the lowfrequency optical conductivity shown in Fig. 2a (see the supplemental materials for additional information). Here, we see good agreement between the experimental $E_{\text {g,opt }}$ value of lightly doped $\mathrm{SnSe}$ and the theoretically predicted $E_{g, o p t}$ value (for comparison of the band separation, $\Delta$, see the supplemental materials, Fig. S10). This demonstrates that the lightly doped sample captures the genuine electronic structure of $\mathrm{SnSe}$. 


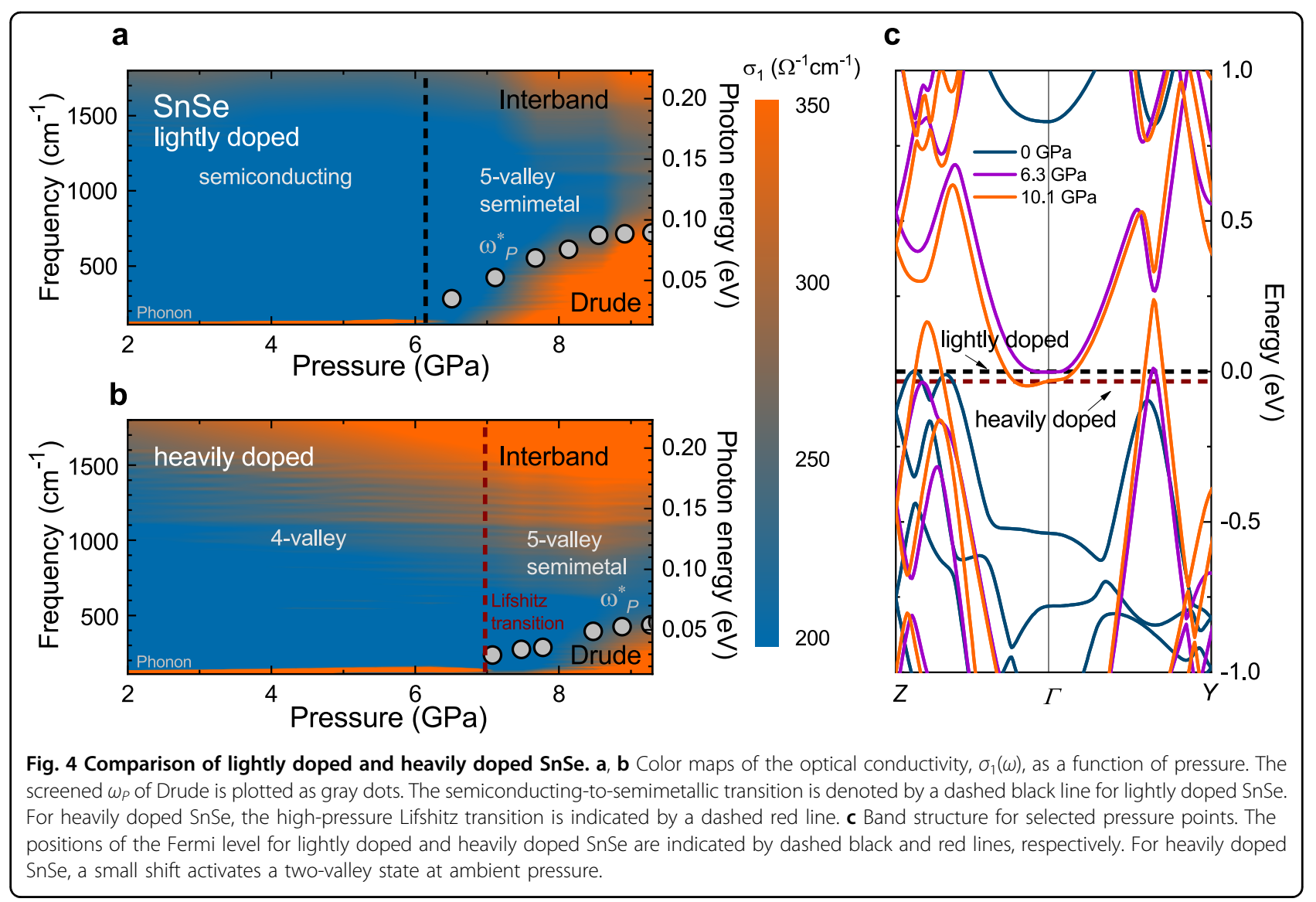

\section{Effect of self-doping under pressure}

The differences between lightly doped and heavily doped SnSe are assumed to originate from a small shift of the Fermi level toward the valence band ${ }^{17,24}$. This shift results in qualitatively and quantitatively different features under pressure as well as distinct resistivity at ambient pressure (see Fig. 1c for the resistivity). In the contour plots in Fig. 4a, b, we compare the evolution of the lowfrequency electronic structure of both samples under pressure. At low pressure, the optical conductivity possesses a phonon mode at $120 \mathrm{~cm}^{-1}$, which disappears as the Drude response becomes dominant, converting the system into a semimetallic phase (above $6 \mathrm{GPa}$ ). Increasing the pressure strengthens the Drude mode as the number of charge carriers increases owing to Fermi surface expansion. At the same time, narrowed interband transitions smear into the low-frequency spectra, increasing their contributions.

Heavily doped SnSe shows a weaker and delayed Drude response (above $7 \mathrm{GPa}$ ), as indicated by the SW (Fig. 2c) and the plasma frequencies, $\omega_{P}$, obtained by a DrudeLorentz fit (see the supplemental materials). These observations confirm that the Fermi level shifts toward lower energy, explaining the wider optical gap in the heavily doped sample that causes a larger Moss-Burstein contribution, $\Delta E_{g}^{B M}$. Furthermore, we note that above $10 \mathrm{GPa}$, the band structure changes only slightly until $14 \mathrm{GPa}$ (Fig. S11 and Fig. S12). In fact, a shift in the Fermi level for heavily doped samples activates the valence band maximum in the $\Gamma-Z$ direction at ambient pressure, as shown in Fig. 4c, causing metallic-like resistivity behavior (Fig. 1c). However, we note that the resistivity of the heavily doped sample is still relatively high, whereas the contribution to the optical conductivity is barely observable $\left(\sigma_{0} \approx 12 \Omega^{-1} \mathrm{~cm}^{-1}\right)$. The $\Gamma-Y$ direction is further involved at lower pressures, generating an additional hole pocket. This low-pressure transition is suggested by our DFT calculations, in accordance with previously mentioned transport studies, suggesting a Lifshitz transition at $0.86 \mathrm{GPa}^{27}$ from a two-valley system to a four-valley system. We note that in the present study, the low-energy limitations of high-pressure optical measurements mask the appearance of the low-pressure Lifshitz transition, whose carrier density is expected to be too small to be detected in our experiment. Importantly, a shift in the Fermi energy causes the conduction band minimum at the $\Gamma$ point to touch $E_{F}$ at higher pressures (above $7 \mathrm{GPa}$ ) in heavily doped $\mathrm{SnSe}$, creating an additional electron pocket, which is well pronounced in our experimental spectra. This electron pocket further changes the topology of the 
Fermi surface; that is, a Lifshitz transition occurs. Thus, heavily doped SnSe undergoes successive Lifshitz transitions below $1 \mathrm{GPa}$ and above $7 \mathrm{GPa}$, as shown in Fig. $1 \mathrm{~d}$.

Pressure-induced metallization of SnSe is observed by transport measurements, but the transition pressure is different ${ }^{33,34}$. This discrepancy may be caused by variations in the definition of the semiconductor-semimetal transition pressure and differences between powder and single-crystalline samples ${ }^{33}$. Furthermore, we demonstrated that self-doping effects strongly influence the pressure evolution.

\section{Discussion}

For both samples, the semimetallic high-pressure phase originates from a change in the atomic coordinate $z$ of the Sn atom along the $c$ axis. Although the sample remains in the Pnma space group ${ }^{31,34,47}$, the $c$ axis shows more drastic compression than the other axes ${ }^{47}$. In particular, within the scope of the calculations, only adjusting the lattice parameter does not lead to a metallic phase, even up to $10 \mathrm{GPa}$. Therefore, we suggest that the previously reported decrease in the $\mathrm{Sn} z$-parameter ${ }^{47}$ is a crucial factor driving the system into a metallic phase. As previously mentioned ${ }^{36}$, pressure affects the $\mathrm{SnSe}$ bonds along the $c$ axis, leading to hybridization of $\mathrm{Sn}-5 s, \mathrm{Sn}-5 p$, and Se- $4 p$ orbitals, thus opening an additional conduction channel. In this system, for heavily doped SnSe, the $c$-direction (armchair) is conductive, followed by the $b$-direction (zigzag), ultimately inducing a metallic intralayer state.

The shift in the Fermi level of heavily doped samples, however, crucially changes the pressure dependence. In fact, the successive Lifshitz-type transitions in heavily doped $\mathrm{SnSe}$ are distinct from semiconductor-semimetal transitions and the mere creation of a Fermi surface in the lightly doped samples. Owing to the lowered Fermi level, the creation of the electron pocket at the $\Gamma$ point takes place at a higher pressure, expanding the already existing Fermi surface of hole pockets in heavily doped SnSe.

The multivalley physics induced by the application of pressure could also play a role in thermoelectric devices since the effective mass is affected by the number of activated valleys in a material ${ }^{8}$. The high thermoelectric properties reported in Ref. ${ }^{27}$ prove the effects of the multivalley SnSe. However, as the thermoelectric efficiency is determined by a combination of several competing parameters, the effects of an additional electron pocket on the thermoelectric properties remain to be clarified in other studies.

The modified contributions of the $\alpha$ - and $\beta$ transitions to the optical gap further offer unique tunability and accessibility to the valley degree of freedom. In particular, with the application of pressure, we can tune the system from a dichroic crystal to a non-dichroic crystal (when $\alpha$ and $\beta$ become comparable). Such a property opens a promising route to valleytronics ${ }^{1-3}$.

In summary, we probed the electronic multivalley band structure of SnSe over a broad pressure range by optical spectroscopy. By combining our spectroscopic investigation with DFT calculations, we clarified the semiconductorsemimetal transition in lightly doped $\mathrm{SnSe}$ and observed successive Lifshitz transitions in heavily doped samples. We emphasized that self-doping has a considerable influence on the optical properties of SnSe by shifting the Fermi level, which is more pronounced under applied pressure. Our spectroscopic analysis traced the electronic structure evolution under pressure and allowed us to suggest possible applications in the fields of optoelectronics and thermoelectrics by demonstrating versatile tunability under pressure.

\section{Acknowledgements \\ We thank Artem Pronin for the fruitful discussion and Gabriele Untereiner for} continuous experimental support.

\section{Author details}

${ }^{1} 1$. Physikalisches Institut, Universität Stuttgart, Stuttgart, Germany. 2Experimental Physics VI, Center for Electronic Correlations and Magnetism, University of Augsburg, Augsburg, Germany. ${ }^{3}$ Computer, Electrical, and Mathematical Sciences and Engineering Division, King Abdullah University of Science and Technology (KAUST), Thuwal, Saudi Arabia. ${ }^{4}$ Center for Condensed Matter Sciences and Center of Atomic Initiatives For New Materials, National Taiwan University, Taipei, Taiwan

\section{Funding}

The project was supported by the Deutsche Forschungsgemeinschaft (DFG) through grant DR228/52-1. E.U. acknowledges the European Social Fund and the Baden-Württemberg Stiftung for the financial support of this research project by the Eliteprogramm. Open Access funding enabled and organized by Projekt DEAL.

\section{Conflict of interest}

The authors declare that they have no conflict of interest.

\section{Publisher's note}

Springer Nature remains neutral with regard to jurisdictional claims in published maps and institutional affiliations.

Supplementary information The online version contains supplementary material available at https://doi.org/10.1038/s41427-021-00283-2.

Received: 30 April 2020 Revised: 23 November 2020 Accepted: 14 December 2020.

Published online: 5 February 2021

\section{References}

1. Schaibley, J. R. et al. Valleytronics in 2D materials. Nat. Rev. Mater. 1, 16055 (2016).

2. Behnia, K. Polarized light boosts valleytronics. Nat. Nanotechnol. 7, 488-489 (2012).

3. Lin, S. et al. Accessing valley degree of freedom in bulk Tin (II) sulfide at room temperature. Nat. Commun. 9, 1455 (2018).

4. Rycerz, A., Tworzydlo, J. \& Beenakker, C. W. J. Valley filter and valley valve in graphene. Nat. Phys. 3, 172-175 (2007).

5. Li, H.-K. et al. Valley optomechanics in a monolayer semiconductor. Nat. Photon. 13, 397-401 (2019). 
6. Zhao, L.-D. et al. Ultrahigh power factor and thermoelectric performance in hole-doped single-crystal SnSe. Science 351, 141-144 (2016).

7. Zhang, J. et al. Discovery of high-performance low-cost n-type Mg3Sb2-based thermoelectric materials with multi-valley conduction bands. Nat. Commun. $\mathbf{8}$ 13901 (2017)

8. Snyder, G. J. \& Toberer, E. S. Complex thermoelectric materials. Nat. Mater. 7, 105-114 (2008).

9. Pei, Y., Wang, H. \& Snyder, G. J. Band engineering of thermoelectric materials. Adv. Mater. 24, 6125-6135 (2012)

10. Xin, J. et al. Valleytronics in thermoelectric materials. npj Quant. Mater. 3, 9 (2018).

11. Zhao, L.-D. et al. Ultralow thermal conductivity and high thermoelectric figure of merit in SnSe crystals. Nature 508, 373-377 (2014).

12. Li, C. W. et al. Orbitally driven giant phonon anharmonicity in SnSe. Nat. Phys 11, 1063-1069 (2015).

13. Liu, F. et al. Phonon anharmonicity in single-crystalline SnSe. Phys. Rev. B 98, 224309 (2018)

14. Skelton, J. M. et al. Anharmonicity in the high-temperature $\mathrm{Cmcm}$ phase of SnSe: soft modes and three-phonon interactions. Phys. Rev. Lett. 117, 075502 (2016).

15. Chang, C., Tan, G., He, J., Kanatzidis, M. G. \& Zhao, L.-D. The thermoelectric properties of SnSe continue to surprise: extraordinary electron and phonon transport. Chem. Mater. 30, 7355-7367 (2018).

16. Lu, Q. et al. Unexpected large hole effective masses in SnSe revealed by angle-resolved photoemission spectroscopy. Phys. Rev. Lett. 119, 116401 (2017).

17. Wang, Z. et al. Defects controlled hole doping and multivalley transport in SnSe single crystals. Nat. Commun. 9, 47 (2018).

18. Tayari, V. et al. Quasi-two-dimensional thermoelectricity in SnSe. Phys. Rev. B 97, 045424 (2018)

19. Maeda, M. et al. Unusually large chemical potential shift in a degenerate semiconductor: Angle-resolved photoemission study of SnSe and Na-doped SnSe. Phys. Rev. B 97, 121110(R) (2018).

20. Pletikosić, I. et al. Band structure of the IV-VI black phosphorus analog and thermoelectric SnSe. Phys. Rev. Lett. 120, 156403 (2018).

21. Shi, G. \& Kioupakis, E. Anisotropic spin transport and strong visible-light absorbance in few-layer SnSe and GeSe. Nano Lett. 15, 6926-6931 (2015).

22. Singh, N. K. et al. The effect of doping on thermoelectric performance of $p$ type SnSe: Promising thermoelectric material. J. Alloy. Compd. 668, 152-158 (2016).

23. He, W. et al. High thermoelectric performance in low-cost SnS0.91Se0.09 crystals. Science 365, 1418-1424 (2019).

24. Wei, P.-C. et al. Thermoelectric figure-of-merit of fully dense single-crystalline SnSe. ACS Omega 4, 5442-5450 (2019).

25. Mao, H.-K., Chen, X.-J., Ding, Y., Li, B. \& Wang, L. Solids, liquids, and gases under high pressure. Rev. Mod. Phys. 90, 015007 (2018)

26. Lifshitz, I. M. Anomalies of electron characteristics of a metal in the high pressure region. Sov. Phys. JETP 11, 1130-1135 (1960).

27. Nishimura, T. et al. Large enhancement of thermoelectric efficiency due to a pressure-induced lifshitz transition in SnSe. Phys. Rev. Lett. 122, 226601 (2019).
28. Mori, H., Usui, H., Ochi, M. \& Kuroki, K. Temperature- and doping-dependent roles of valleys in the thermoelectric performance of SnSe: a first-principles study. Phys. Rev. B 96, 085113 (2017).

29. Zhang, Y., Hao, S., Zhao, L.-D., Wolverton, C. \& Zeng, Z. Pressure induced thermoelectric enhancement in SnSe crystals. J. Mater. Chem. A 4, 12073-12079 (2016).

30. Yu, H., Dai, S. \& Chen, Y. Enhanced power factor via the control of structural phase transition in SnSe. Sci. Rep. 6, 26193 (2016).

31. Efthimiopoulos, l. et al. Effects of temperature and pressure on the optical and vibrational properties of thermoelectric SnSe. Phys. Chem. Chem. Phys. 21 8663-8678 (2019).

32. Su, N. et al. Pressure-induced enhancement of thermoelectric power factor in pristine and hole-doped SnSe crystals. RSC Adv. 9, 26831-26837 (2019).

33. Yan, J. et al. Pressure-driven semiconducting-semimetallic transition in SnSe Phys. Chem. Chem. Phys. 18, 5012-5018 (2016).

34. Chen, $X$. et al. Topological Dirac line nodes and superconductivity coexist in SnSe at high pressure. Phys. Rev. B 96, 165123 (2017).

35. Ghosh, A. et al. Electrical properties of SnSe under high-pressure. Comput. Condens. Matter 9, 77-81 (2016).

36. Peng, J. et al. Pressure-induced improvement in symmetry and change in electronic properties of SnSe. J. Mol. Model. 23, 319 (2017).

37. Dewaele, A., Torrent, M., Loubeyre, P. \& Mezouar, M. Compression curves of transition metals in the Mbar range: experiments and projector augmentedwave calculations. Phys. Rev. B 78, 104102 (2008).

38. Datchi, F. et al. Optical pressure sensors for high-pressure-high-temperature studies in a diamond anvil cell. High. Press. Res. 27, 447-463 (2007).

39. Look, D. C. \& Leach, J. H. On the accurate determination of absorption coefficient from reflectance and transmittance measurements: application to Fe-doped GaN. J. Vac. Sci. Technol. B 34, 04J105 (2016).

40. Tanner, D. B. Optical Effects in Solids (Cambridge University Press, 2019).

41. Basov, D. N., Averitt, R. D., van der Marel, D., Dressel, M. \& Haule, K. Electrodynamics of correlated electron materials. Rev. Mod. Phys. 83, 471-541 (2011)

42. Dressel, M. \& Grüner, G. Electrodynamics of Solids: Optical Properties of Electrons in Matter (Cambridge University Press, 2002).

43. Blaha, P et al. WIEN2k. An Augmented Plane Wave + Local Orbitals Program for Calculating Crystal Properties (Karlheinz Schwarz, Techn. Universität Wien, Austria), 2018. ISBN 3-9501031-1-2.

44. Tran, F. \& Blaha, P. Accurate band gaps of semiconductors and insulators with a semilocal exchange-correlation potential. Phys. Rev. Lett. 102, 226401 (2009).

45. Koller, D., Tran, F. \& Blaha, P. Improving the modified Becke-Johnson exchange potential. Phys. Rev. B 85, 155109 (2012).

46. Perdew, J. P., Burke, K. \& Ernzerhof, M. Generalized gradient approximation made simple. Phys. Rev. Lett. 77, 3865-3868 (1996).

47. Loa, I., Husband, R. J., Downie, R. A., Popuri, S. R. \& Bos, J.-W. G. Structural changes in thermoelectric SnSe at high pressures. J. Phys. Condens. Matter 27, 072202 (2015).

48. Burstein, E. Anomalous optical absorption limit in InSb. Phys. Rev. 93, 632-633 (1954).

49. Moss, T. S. The interpretation of the properties of indium antimonide. Proc. Phys. Soc. B 67, 775-782 (1954). 